MODELING, IDENTIFICATION AND CONTROL, 1984, VOL. 5, NO. 2, 123-128

doi:10.4173/mic.1984.24

\title{
Optimal control of an offshore lifting operation
}

\author{
K. M. MJELDE†, P. C. S. LEE, W. QVAM, H. P. JACOBSEN, \\ P. ROONEY and F. TEGN
}

Keywords: Safety analysis, linear programming, optimal control.

\begin{abstract}
The optimal control of an offshore operation is analysed: a hideck which is at rest on a barge is lifted from the barge by means of a partly submerged frame that receives buoyancy by pumping water out of three cylinders connected to the frame. Air is pumped into the cylinders in order to maintain a positive difference between the internal air-pressure and the external water-pressure: for any cylinder, this pressure-difference is possibly critical at certain regions located on the cylinder and on a spherical cupola on the top of it. Optimal pumping rates of water and air as a function of time are determined, such as to minimize the maximum of certain weighted pressure differences at the critical regions, and such as to satisfy given geometrical and dynamical boundary conditions.
\end{abstract}

\section{Introduction}

For the purpose of the analysis described in this paper the system considered consists of three parts: a hideck, a frame and three cylinders connected to the frame; each cylinder has a spherical cupola (see the Figure). The cylinders are filled with water and air.

Initially the hideck is at rest on a barge and the frame is in forceless contact with the hideck: the barge being located within a slot in the frame. The hideck is then lifted from the barge by pumping water out of the cylinders. The lifting operation terminates when the hideck is just out of contact with the barge, and the hideck rests on the frame at zero vertical velocity, so that the frame will not come in contact with the barge. The pressure inside a cylinder is controlled by air compressors; the airpressure is possibly critical along two circles: the external overpressure must not be too high along the circle determined by the water-level inside a cylinder; the internal overpressure is critical along a certain circle located on the cupola.

The purpose of this paper is to describe a model for the determination of pumping rates of water and air that executes the previously described lifting operation in such a way that the danger is minimized of the collapse of a cylinder or a cupola along one of the previously described critical circles.

The model was applied to check the operation of the mating of a hideck and a Technomare Steel Gravity frame within the offshore development scheme for the Maureen field. Although several aspects of the Maureen concept have been considered in the literature, see for instance Bilderbeck (1982) and Gotsill (1982), a mathematical

Received 20 September 1983.

+ Det Norske Veritas, Veritasveien 1, N-1322 Høvik, Norway. 


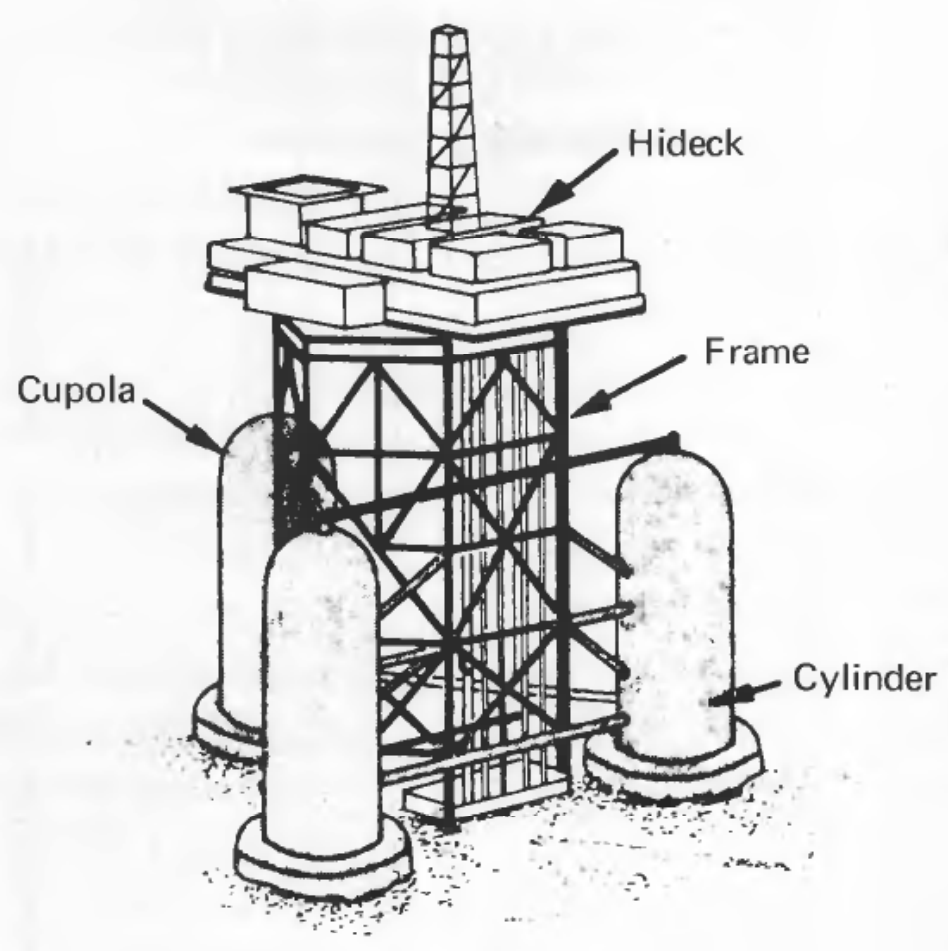

The system.

programming model for the analysis of the safety of the platform during marine operations has not previously been published.

\section{The model}

Although the motion of the hideck is extremely slow the operation is analysed in terms of a set of dynamical equations, rather than in terms of a sequence of equilibrium states. This is due to the fact that the slow motion comes from the large total vass of the system and the relatively small forces involved, as obtained by pumping water and air in and out of the cylinders. Furthermore, it is necessary to control the pumping rates and the associated dynamical forces such that the velocity of the frame is zero when the hideck is lifted out of contact with the barge.

A planning period of length $T$ is selected, and divided into $N$ subperiods of lengths $\Delta T=T \mid N$. The cylinders are assumed to move in parallel and the state of the system is defined by:

$x_{n}$ : The position of a cylinder, given by the distance from the surface of the water to the bottom of the cylinder at time $n \Delta T$ for $n=0, \ldots, N$, measured positive downwards.

$u_{n}$ : The velocity of a cylinder at time $n \Delta T$ for $n=0,1, \ldots, N$, measured positive downwards.

$y_{i n}$ : The increase in the quantity of water $(i=1)$ and air $(i=2)$ in a cylinder during the time interval $[0, n \Delta T]$; for $n=1, \ldots, N$. 
Since the total initial force on the cylinders is zero, the kinematic equations are given as follows:

$$
\begin{gathered}
x_{n}=x_{n-1}+u_{n} \Delta T ; n=1, \ldots, N \\
u_{n}=u_{n-1}+\frac{1}{M_{n}}\left[g\left(y_{1 n}+y_{2 n}\right)+F_{1}\left(x_{0}, x_{n}\right)+F_{2}\left(x_{0}, x_{n}\right)\right] ; n=1, \ldots, N
\end{gathered}
$$

with the boundary conditions:

$$
\begin{aligned}
x_{0}=a_{0}, & u_{0}=0 \\
x_{N}=a_{N}, & u_{N} \in[-\epsilon, 0] \\
x_{n-1}>x_{n} ; & n=1, \ldots, N
\end{aligned}
$$

where $a_{0}$ and $a_{N}$ are given and $\epsilon>0$ is a small number (since a velocity that is exactly zero at time $N \Delta T$ may be difficult to achieve).

The constraint (5) requires a motion in one direction; in particular, $x_{n} \geqslant x_{N}$ for all $n=1, \ldots, N$ and there is no terminal oscillations.

The physical interpretations are as follows:

$M_{n}$ : The mass at time $n \Delta T$ of the system in motion

$g$ : Gravity acceleration

$F_{i}\left(x_{0}, x_{n}\right)$ : The decrease of the buoyancy force of the frame at the top of the cylinders $(i=1)$ and of the barge $(i=2)$ when the position of the cylinders changes from $x_{0}$ to $x_{n}$; the force is positive downwards.

The mass $M_{n}$ is expressed as follows:

where

$$
M_{n}=M+m_{10}+m_{20}+y_{1 n}+y_{2 n}+G+G_{B}
$$

$M$ : The mass of a cylinder plus one third of the mass of the frame.

$m_{i 0}$ : The initial quantity of water $(i=1)$ and air $(i=2)$ in a cylinder.

$G$ : One third of the mass of the hideck.

$G_{B}$ : One third of the mass of the barge.

The initial forces on a cylinder at rest have a zero resultant, and since the cylinders are submerged during the whole operation the only forces that change are the buoyancy force of the frame; the weights of water and air; and the lifting force exerted on the hideck.

The motion of the cylinders is controlled by the following decision variables:

$\phi_{i n}$ : The rate of increase of the mass of water $(i=1)$ and of air $(i=2)$ in the time interval $[(n-1) \Delta T, n \Delta T]$, as determined by the application of pumps, compressors and valves.

It follows that:

where

$$
y_{i n}=\sum_{j=1}^{n} \phi_{i j} \Delta T ; \quad n=1, \ldots, N ; \quad i=1,2
$$

and

$$
\underline{b}_{i n} \leqslant \phi_{i n} \leqslant \bar{b}_{i n} ; \quad n=1, \ldots, N ; \quad i=1,2 .
$$

$$
\underline{b}_{i n} \leqslant 0, b_{i n} \geqslant 0 ; \quad n=1, \ldots, N ; \quad i=1,2
$$


The requirement $\underline{b}_{i n}=\bar{b}_{i n}=0$ for a $n \in\{1, \ldots, N\}$ demands that $\phi_{i n}=0$, as may be required for monitoring checking during the time interval $[(n-1) \Delta T, n \Delta T]$.

The difference between the internal and the external pressures must be within given bounds at certain critical circles on the cylinders, and the following quantities are defined accordingly:

$\Delta_{1 n}$ : The pressure difference at the internal water-line of a cylinder (external overpressure critical)

$\Delta_{2 n}$ and $L$ : The pressure difference along the circle determined by the horizontal plane a distance $L$ from the top of the cupola of a cylinder (internal overpressure critical).

With

$$
p_{2 n}=\frac{\frac{p_{20}}{\rho_{20}}\left[m_{20}+y_{2 n}\right]}{V-\frac{m_{10}+y_{1 n}}{\rho_{10}}}
$$

it follows that

$$
\Delta_{1 n}=p_{2 n}-P_{0}-\rho_{10}\left[x_{n}-\frac{m_{10}+y_{1 n}}{\rho_{10} A}\right] ; n=1, \ldots N
$$

and

$$
\Delta_{2 n}=p_{2 n}-P_{0}-\rho_{10}\left[x_{n}-L\right] ; n=1, \ldots, N
$$

where the definitions of quantities are as follows:

$p_{20}$ : The air-pressure inside a cylinder in the initial condition (at rest in position $x_{0}$ )

$\rho_{20}$ : The initial density of the air in a cylinder

$\rho_{10}$ : The density of water (assumed to be identical inside and outside a cylinder, corrections for different densities are obvious)

$V:$ The internal volume of a cylinder

$P_{0}$ : The atmospheric pressure

$A$ : The internal cross sectional area of a cylinder

The mathematical problem, denoted by $Q$, is to determine the controls $\phi_{\text {in }}$ for $i=1,2$ and $n=1, \ldots, N$ such as to minimize the maximum of the weighted overpressures:

$$
Q: \text { Minimize } z=\operatorname{Max}_{n=1, \ldots, N}\left[-\Delta_{1 n} \gamma_{1}, \Delta_{2 n} \gamma_{2}\right]
$$

subject to the constraints:

$$
\begin{aligned}
-\Delta_{1 n} & \leqslant \bar{p}_{1} \\
\Delta_{2 n} & \leqslant \bar{p}_{2}
\end{aligned}
$$

and the constraints and initial conditions $(1,2,3,4,5,7,8,9)$.

The numbers $\bar{p}_{1}$ and $\bar{p}_{2}$ are given values equal to the upper bounds of the external overpressure $-\Delta_{1 n}$ and the internal overpressure $\Delta_{2 n}$, and the numbers $\gamma_{1}$ and $\gamma_{2}$ may be chosen such that $\gamma_{i}=1 / \bar{p}_{t}$ for $i=1,2$, in order to reflect the relative sizes of the allowable pressures in the objective function. 


\section{Linear programming approximation}

In order to solve the problem by linear programming, certain approximations have to be introduced.

In eqn. (2) the change $F_{1}\left(x_{0}, x_{n}\right)$ of the buoyancy of the frame is small compared to the change $F_{2}\left(x_{0}, x_{n}\right)$ of the buoyancy of the barge, the latter quantity being given by a linear expression. Consequently, in eqn. (2):

$$
\begin{aligned}
& F_{1}\left(x_{0}, x_{n}\right)=0 \\
& F_{2}\left(x_{0}, x_{n}\right)=\rho_{10} A_{B}\left(x_{0}-x_{n}\right) g
\end{aligned}
$$

where:

$A_{B}$ : the cross sectional area of the barge along the water line (nearly constant).

Since $\left(y_{1 n}+y_{2 n}\right)$ is small compared to the other terms in the expression for $M_{n}$, linearization of the right hand side of eqn. (2) by expansion in a Taylor series, is approximately equal to the substitution of $y_{1 n}=y_{2 n}=0$ in the expression for $M_{n}$ in eqn. (2); this approximation is introduced.

The replacement of the expression for $p_{2 n}$ in eqns. (11) and (12) by a Taylor-series linearization, makes it possible to approximate the problem $Q$ by a linear programming problem, denoted by $Q A$, as follows:

$Q A:$ Minimize $z=\lambda$

subject to the constraints:

$$
\begin{array}{ll}
\lambda \geqslant-\Delta_{1 n} \gamma_{1} ; & n=1, \ldots, N \\
\lambda \geqslant \Delta_{2 n} \gamma_{2} ; & n=1, \ldots, N
\end{array}
$$

and the previously given constraints $(1,2,3,4,5,7,8,9,14,15)$, with the above approximations introduced in the expressions for $M_{n}, F_{1}\left(x_{0}, x_{n}\right)$ and $p_{2 n}$ for $n=1, \ldots, N$.

Additional constraints may be introduced in the problem $Q A$ by a trial and error procedure, or explicitly. For instance, a control plan with $\phi_{1 n} \cdot \phi_{2 n}=0$ for $n-1, \ldots, N$ may be checked by assuming given values of $\phi_{1 n}$ for $n=1, \ldots, N$, and calculating the corresponding optimal values of $\phi_{2 n}$ for $n=1, \ldots, N$; or conversely, $\phi_{2 n}$ for $n=1, \ldots, N$ are given and $\phi_{1 n}$ for $n=1, \ldots, N$ are calculated, the result from each optimization being applied to indicate changes of the allocation plan.

The previously given linear approximations are applicable to the control of the remaining motion of the system, when the system is at any intermediate state, as identified by the instruments on the hideck; the quality of the linear approximations has a tendency to improve towards the end of the lifting operation.

By observing the number of equality constraints in the linear programming problem, and comparing this number with the total number of variables in the problem, it follows that a large number of the controls $\phi_{1 n}$ and $\phi_{2 n}$ for $n=1, \ldots, N$ is expected to be bang-bang controls (taking their extreme values), as confirmed by numerical computations.

Finally, it is noted that failure probabilities $f_{1 n}\left(-\Delta_{1 n}\right)$ and $f_{2 n}\left(\Delta_{2 n}\right)$ can be introduced in the model by the application of the bounded variable method of Dantzig (1963), provided the functions $f_{1 n}\left(-\Delta_{1 n}\right)$ and $f_{2 n}\left(\Delta_{2 n}\right)$ are concave for $n=1, \ldots, N$; in such a model the maximal failure probability is minimized. 


\section{Comments}

During the lifting operation, a cylinder was demonstrated to be safe against collapse failure along the inside water level with a considerable safety margin. The critical region is the circle along the intersection between a cupola and a cylinder, where an inside overpressure with respect to the surrounding hydrostatic pressure is to be expected. This indicated that adequate air compression to the cylinders should be pursued during the operation.

Theoretical solutions suggested that one can adopt an operation with uniform pumping rates with pauses for monitoring checks.

\section{REFERENCES}

BILDERBECK, M. G. (1982). Innovations help develop marginal North Sea field, Oil and Gas, June,

Gotsill, A. (1982). Hunterston float-out marks Maureen milestone, review, Offshore Engineer, November,

DANTZIG, G. B. (1963). Linear programming and extensions, Princeton University Press; Princeton, New Jersey. 